\title{
A Dynamic Clustering Approach for Maximizing Scalability in Wireless Sensor Networks
}

\author{
${ }^{1}$ Hanane Kalkha, ${ }^{1,2}$ Hassan Satori, ${ }^{1}$ Khalid Satori \\ ${ }^{1}$ Department of Computer Science, \\ Faculty of sciences Dhar el Mahrez, USMBA \\ Fez, Morocco \\ ${ }^{2}$ Department of Mathematics and Computer Science \\ Multidisciplinary Faculty, Mohamed First University \\ Nador, Morocco \\ hanane.kalkha@usmba.ac.ma; hsatori@yahoo.com
}

\begin{abstract}
Scalability is an important and crucial issue which in routing protocols for Wireless Sensor Networks (WSNs). In this paper, we present an approach to achieving a balanced energy consumption rate using dynamic clustering to provide scalability in WSN. The proposed work in this paper is based on the dynamic clustering using k-means compared to LEACH (Low-Energy Adaptive Clustering Hierarchy), which is one of the most simple and effective clustering solutions widely deployed for WSN. The simulation results show that our proposed algorithm significantly improves high network scalability compared to LEACH.

Keywords- WSN; energy-efficient; K-means; LEACH; clustering;
\end{abstract}

\section{Introduction}

Wireless Sensor Network (WSN) is a popular research area, due to the potential use of sensor networks in different fields. A sensor network is a compound, of sensing, processing, communication ability to observe and react to events in a specified environment. WSN is usually composed of tens to thousands of nodes. These nodes collect, process and transmit cooperatively information to a central location. WSNs have specific problems such as a low duty cycle, power and life limited battery constraints, the acquisition of redundant data, heterogeneous sensor nodes, node mobility, and dynamic network topology, etc. [1, 2].

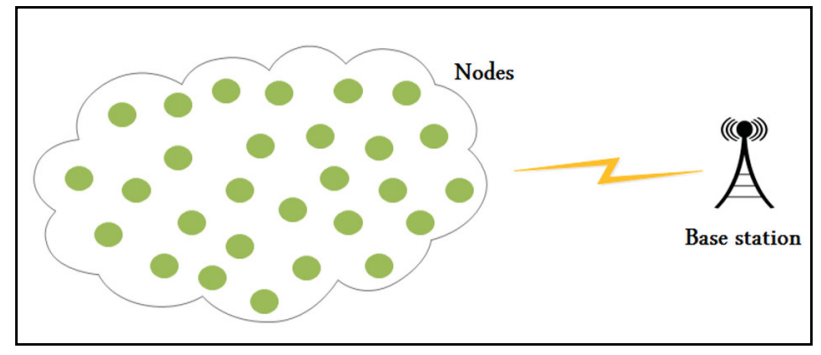

Fogure 1 Wireless sensor network architecture 
Hanane Kalkha, Hassan Satori, Khalid Satori. A Dynamic Clustering Approach for Maximizing Scalability in Wireless Sensor Networks. Transactions on Machine Learning and Artificial Intelligence, Vol 5 No 4 August (2017); p: 637-650

WSN has interesting applications such as environmental monitoring and tracking applications, this was approved recently by the availability of smaller, cheaper, and intelligent sensors. These sensors have limited power resources generally irreplaceable. Therefore, when the ordinary networks aim to attain a high quality of service (QOS), WSN should focus first on the energy conservation. So, they must have integrated mechanisms that compromise offer the possibility to extend the network life at the cost of lower throughput and high transmission delay [3, 4].

Hence, the design energy-aware algorithms become an important factor to extend the sensor lifetime. Grouping nodes in clusters were widely followed by researchers to achieve the network scalability objective. Each group will have a chief often called the cluster head (CH) [5]. While many classification algorithms have been proposed in the literature for ad hoc networks. The majority of these algorithms is concerned mostly about the node accessibility and stability of the route without worrying about the critical design sensor networks [6]. Recently, several clustering algorithms have been specially adapted for sensor networks [7]. These available classification techniques varied widely according to the numbers of nodes, the network architecture, the characteristics of $\mathrm{CH}$ nodes and network process model. A CH can be elected by the sensors into a cluster or pre-allocated by the network designer and can also be simply one of the sensors, or a node which is higher in resources. The cluster composition can be fixed or variable. $\mathrm{CHs}$ can provide a second bearing network or may simply send the data to interested parts, for example, a base station or a command center [8].

The primary objective of hierarchical routing is to maintain effectively the energy consumption of sensor nodes by engaging them in multi-hop communication in a specific cluster and performing data aggregation and fusion in order to reduce the number of messages forwarded to the sink. Cluster formation is generally based on the energy reserved of the sensors and detection of cluster head [9]. LEACH protocol (Low Energy Adaptive Clustering Hierarchy) [10] is among the first hierarchical routing approaches for sensor networks. The proposed LEACH concept was an inspiration for many hierarchical routing protocols in the literature.

Even though the LEACH protocol act in a good manner, it also suffers from several disadvantages such: Firstly, The cluster head is generated randomly, so that cannot ensure the uniform distribution of $\mathrm{CHs}$. This selection doesn't take into consideration transmission distance and the residual energy of the nodes, resulting uneven energy consumption for different nodes. Secondly, the high frequencies of re-clustering leads to a waste of energy [11].

The aim of this work is to propose an algorithm to extend the network lifetime. Our proposed algorithm was inspired from LEACH and based on k-means algorithm. The remainder of this paper is structured as follows: Section 2 presents the related work, Section 3 is about hierarchical routing and Section 4 discusses the scalability of WSNs, followed by description of used protocol in section 5 . Section 6 present the simulation setup used. The results and discussions are presented in section 8 before concluding the paper.

\section{Related work}

Limited energy resources of sensor nodes create difficult issues on the improvement of routing protocols for WSN. Clustering is an important mechanism in wireless sensor networks to achieve scalability, reducing energy consumption and have a better network performance. Recently, H. Kalkha et al. [12] have compared in their work the performance of the two most famous routing protocols for wireless sensor 
networks AODV and LEACH the first is plat based protocol and the second is a cluster-based protocol, the results showed that $\mathrm{LEACH}$ is more efficient than AODV for different parameters such as energy consumption, throughput and PDR.

Many research projects explored clustering in WSN from various perspectives. LEACH [10] is the first clustering algorithm has been proposed to reduce energy consumption. In which, the grouping task is set in rotation between the nodes, depending on duration. The data transmission between the $\mathrm{CH}$ and the base station (BS) is done through direct communication.

A modified release of LEACH known as LEACH-C [13] (Leach centralized) uses a centralized cluster formation algorithm to form clusters. Centralized approaches allow having an overall view of the network and therefore identifying the heads and members of the cluster. Even if all the information is available in this sort of approach, the determination of a proper value of the number of clusters is still a hard problem.

HEED (Hybrid, Energy-Efficient Distributed Clustering) [14] extends the basic of LEACH using the residual energy and the degree of node or density as a metric for cluster election in order to achieve the balancing power. It works in multi-hop networks by using adaptive power transmission in the inter-cluster communication.

PEGASIS (Power-Efficient Gathering in Sensor Information System) [15] inside instead of transmitting the packets of many cluster heads as such in LEACH, each node will form a chain structure to the base station through which the data is transmitted to the base station. PEGASIS achieves energy efficiency by forwarding data to one of its neighbor nodes.

\section{Hierarchical Routing}

Hierarchical or cluster-based routing are techniques that provide particular advantages of scalability and efficient communication. The hierarchical routing concept is also used to perform energy-efficient routing in WSNs. In a hierarchical architecture, higher energy nodes may be used to process and deliver the information while the lower energy nodes may be used to perform the detection in the proximity of the target. It means the creation of clusters and assigning special tasks to cluster head can contribute greatly to the overall scalability of the system, the lifetime and energy efficiency. So, hierarchical routing is an effective way to reduce energy consumption in a cluster and performing data aggregation and fusion, to reduce the number of messages transmitted to the sink [17]. In clustering approach, wireless sensor network can be considered of having following parts [18]: A group of nodes named cluster. Member nodes of the cluster. Cluster Head $(\mathrm{CH})$ which is the leader of the cluster is charged for the delivery data from the cluster members to different cluster heads or base station Base Station (BS) which relay between network and end-user.

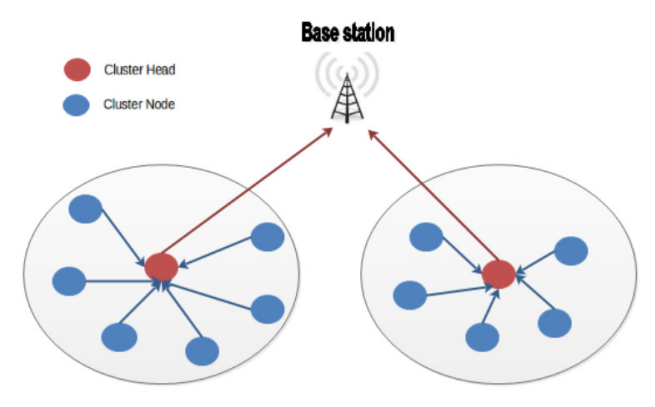

Figure 2 Schematic representation of clustering. 


\subsection{Classification of Clustering Schemes}

Wireless sensor networks clustering schemes may be classified as distributed and centralized. In distributed approach, nodes locally exchange information for selection of $\mathrm{CHs}$ and formation of clusters. In a centralized approach, a central node, such as a base station control selection of $\mathrm{CH}$ s and formation of clusters [18].

For the state of clusters, clustering schemes can be categorized as dynamic clustering and static clustering. In the dynamic clustering, clusters are reformed after the end of the round. In the static grouping, clusters are formed in one and $\mathrm{CHs}$ are selected from cluster nodes.

According to the sensor node's characteristics, clustering algorithms can be classified as heterogeneous clustering and homogenous clustering. In Homogenous clustering the nodes are indistinguishable even there are super nodes. All nodes have equal opportunity to be $\mathrm{CH}$. Heterogeneous clustering algorithms categorize nodes as normal nodes and super nodes. Super nodes are having higher energy than normal nodes and have a high chance to be selected as $\mathrm{CH}$.

\subsection{Dynamic Clustering}

Dynamic clustering is widely used in hierarchical routing, the dynamic clustering technique aim to assign each pattern datum set for the cluster with the closest centroid. The dynamic clustering provides several benefits; For example, clusters are dynamically formed in response to particular triggered events. If a node detects an event with sufficient capacity, the node volunteers to act as a $\mathrm{CH}$. The $\mathrm{CH}$ invites the other nodes to be as members of this cluster. The nodes may be selected on different clusters at different times with different roles [19].

\section{Scalability of WSNs}

Scalability is very important issue in the conception of routing protocols for sensor networks. A routing protocol classed as good and effective when it is adaptable to network topology changes. To determine the scalability in WSN, there are many parameters to consider as the number of nodes, node deployment, time, etc.) [20].

In order to evaluate the scalability of routing protocols, there are many indicators to analyze, such as the network lifetime, throughput and energy consumption, etc. [20]. Figure 3 shows the most important parameters considered in the WSN performance evaluation.

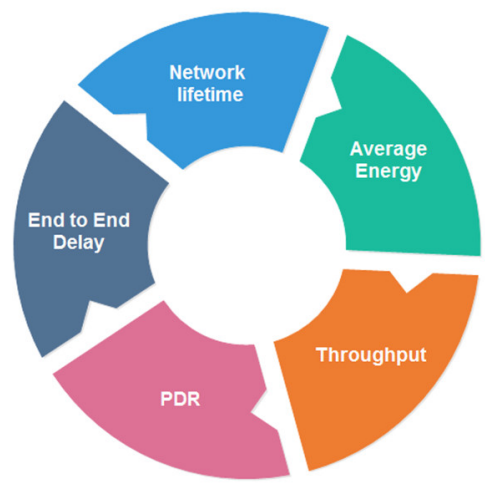

Figure 3 Scalability indicators 


\subsection{Lifetime Network}

The most difficult challenges in the WSN design are how to conserve the node energy without affecting network operation. Moreover, the goal of an energy efficient technique is to maximize network lifetime, which strongly depends on the lifetime of every node.

There are several network lifetime definitions, depending on the wireless sensor network application. The network lifetime is defined in $[21,22]$ as the time until the first node/sensor in the network dies. In contrast, the network lifetime is defined in [10] as the time until all nodes die. The authors of [23] present the networks lifetime as the $\min \{\mathrm{t} 1, \mathrm{t} 2, \mathrm{t} 3\}$, where $\mathrm{t} 1$ is the time it takes for the cardinality of the largest connected components to drop below $\mathrm{c} 1 \cdot \mathrm{n}(\mathrm{t})$, where $\mathrm{n}(\mathrm{t})$ is the number of alive sensors at time $\mathrm{t}$; $\mathrm{t} 2$ is the time it takes for $\mathrm{n}(\mathrm{t})$ to drop below $\mathrm{c} 2 \cdot \mathrm{n}(0)$; and $\mathrm{t} 3$ is the time it takes for the area covered to drop below $\mathrm{c} 3 \cdot \mathrm{A}$, where $\mathrm{A}$ is the area covered by the initial deployment of the nodes. It is well-known that in both the ad-hoc and sensor networks, the network connectivity is very important to ensure the maximal delivery of the collected information [23].

\subsection{Average energy}

Energy consumption is the total energy consumed by the sensor nodes in the network [24], the energy consumption model based on the distance can be represented as follows:

$$
\begin{gathered}
E_{\text {trans }}(n, d)=\left\{\begin{array}{l}
n *\left(E_{\text {elect }}+\varepsilon_{\text {fmp }} * d^{2}\right) d<d_{0} \\
n *\left(E_{\text {elect }}+\varepsilon_{\text {amp }} * d^{2}\right) d \geq d_{0}
\end{array}\right. \\
E_{\text {rec }}=n * E_{\text {elec }}
\end{gathered}
$$

Where,

- $E_{\text {trans }}(n, d)$ :is the data transmit energy consumption,

- $E_{\text {rec: }}$ is the data receiving energy consumption, $n$ is the bit of the data packet.

- Eelec: is the wireless transceiver circuit energy consumption,

- $\varepsilon_{\mathrm{fmp}}$ and $\varepsilon_{\mathrm{amp}}$ : is the amplifier circuit power coefficient for free-space model and multi-path fading model respectively.

- $\mathrm{d}$ : is the distance between the sending node and the receiving node.

The figure below shows the energy model of $L$ bits transmitted over the distance $d$ :

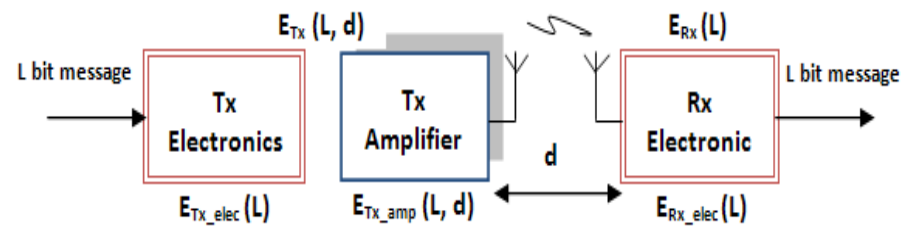

Figure 4 Energy Model in WSN

The equation used to compute average total energy (Eavg) per round is expressed as:

$$
E_{\text {avg }}=\frac{\text { Total energy consumed by all nodes }}{\text { number of nodes }}
$$




\subsection{Throughput}

Throughput is an important metric for improved network protocols, is usually measured in bits per second (bit/s) or sometimes in data packets per second $(\mathrm{p} / \mathrm{s})$ or data packets per time slot. The network throughput is the number of packets received successfully at the destination [25].

\subsection{Packet Delivery Ratio (PDR)}

The ratio of packets which are successfully delivered to a destination compared to the number of packets that were sent by the sender [25].

\section{Description of protocols}

\subsection{LEACH protocol}

Low Energy Adaptive Clustering Hierarchy (LEACH) proposed by W. Heinzelman et. al [10] is a clustering based protocol using the random rotation of local cluster to fairly distribute the energy among the sensors nodes. LEACH uses localized coordination to allow scalability and robustness for dynamic networks. LEACH integrates data fusion in the routing protocol in order to reduce the amount of information required to be sent to the base station. It reorganizes the network clustering dynamically and periodically, this makes it difficult to rely on a long lasting node to node confident link to make the protocol secure. LEACH assumes each node can directly achieve a base station transmitting with sufficiently high power. LEACH consists of two phases: Set-Up Phase and Steady State Phase Figure. 7.

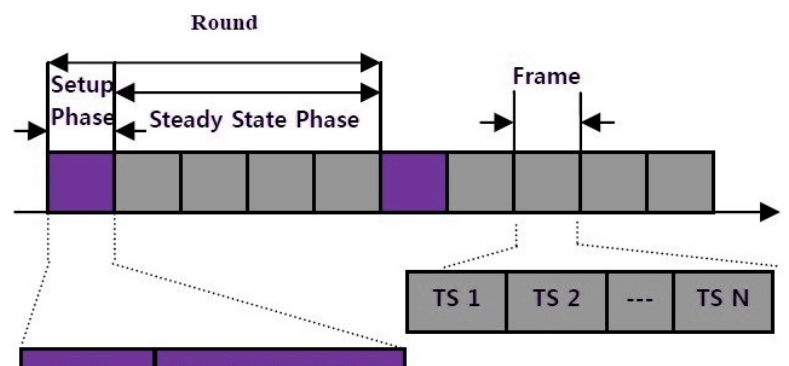

\begin{tabular}{l|l} 
CH Cluster formation & TS: Time Slot
\end{tabular}

Figure 5 LEACH phases.

Set-Up Phase: At first each node computes a single random number between 0 and 1 , and then calculates a threshold of formula $T(n)$.

$$
T(n)=\left\{\begin{array}{lr}
\frac{P}{1-P \times\left(r \bmod \frac{1}{P}\right)} & \text { if }(n \in G) \\
0 & \text { else }
\end{array}\right.
$$

With:

P: percentage of nodes desiring to become cluster-head.

$r$ : current round number.

G: set of nodes that have not been elected cluster-heads during the 1/P last previous periods.

If the random number is less than the calculated threshold the node selected as a cluster head. After its selection each cluster head broadcasts an advertisement message to the rest nodes using the CSMA MAC protocol, and each node selects a cluster head based on the Received Signal Strength Indication (RSSI) of 
the advertisement. Each node uses the CSMA MAC protocol to transmit its selection. After forming clusters, each cluster head create a TDMA schedule based on the number of nodes in the cluster.

Steady State Phase: Each sensor node collects and transmits data to its cluster head based on the TDMA schedule. Cluster heads receive all the data and send it to the base station. After transmission new round starts and executes the setup and steady state phase.

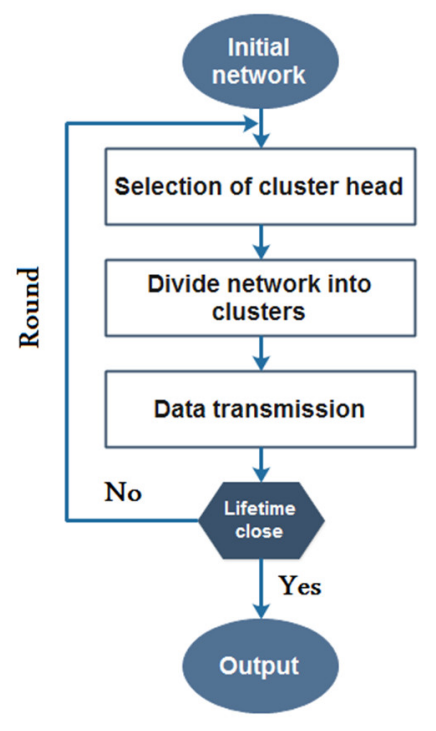

Figure 6 LEACH chart flow.

\subsection{K-MEANS clustering}

$\mathrm{K}$-means algorithm is an unsupervised clustering algorithm, is a widely used technique for clustering based partitioning trying to find a number of specified number of clusters (k), which are represented by their centroid by minimizing the square error function developed for small size data often do not work well for high-dimensional data and the results may not be exact most of the time.

There are two simple approaches to initialize cluster center, i.e. either to choose the initial values randomly, or choose the first $\mathrm{k}$ samples of data points. Alternatively, different sets of initial values are selected (out of the data points) and the set, which is the closest to the optimal, is selected. [26].

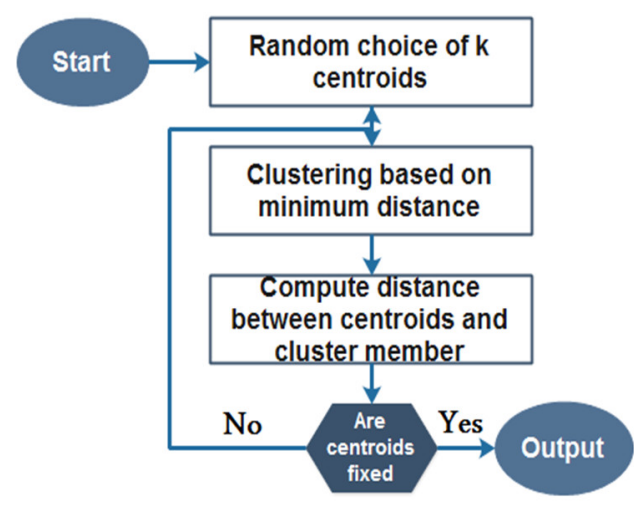

Figure 7 K-means clustering chart flow. 


\subsection{Our proposed algorithm based on K-means}

Our proposed algorithm based on K-means is primarily use the Euclidean distances and selection of the cluster head node depends to residual energies. So, the central node collects the information about the node id, the position and the residual energy of all nodes and stores this information in the central node. After getting this information from all nodes, it starts to perform the clustering algorithm k-means.

\section{Step of clustering}

1. Choose $\mathrm{k}$ sensor nodes from all the $\mathrm{n}$ sensor nodes as the initial data centers.

2. Calculate the Intra-Cluster Distance [20] between other nodes to each of the centers using Euclidean distance as follows (1), and assign each node to the closest center.

$$
\text { intra }=\frac{1}{N} \sum_{i=1}^{k} \sum_{x \in C_{i}}\left\|x-Z_{i}\right\|
$$

$\mathrm{N}$ is the number of nodes in the network,

$\mathrm{K}$ is the number of clusters,

$\mathrm{Zi}$ is the cluster center of cluster $\mathrm{Ci}$.

3. Then assign other nodes to these clusters.

4. And calculate each node's cost. Node having the lowest cost is selected as cluster head.

This cycle continues until the $\mathrm{K}$ cluster heads fix down.

\section{Data Transmission}

Once the clusters are formed and the cluster head is selected.

1. The cluster head will specifies timeslot for each cluster member. Each cluster member transmits the data only in its timeslot.

2. The cluster head collects the data from the cluster member and forwards it to the sink node.

After data transmission, the network chooses the cluster head again.

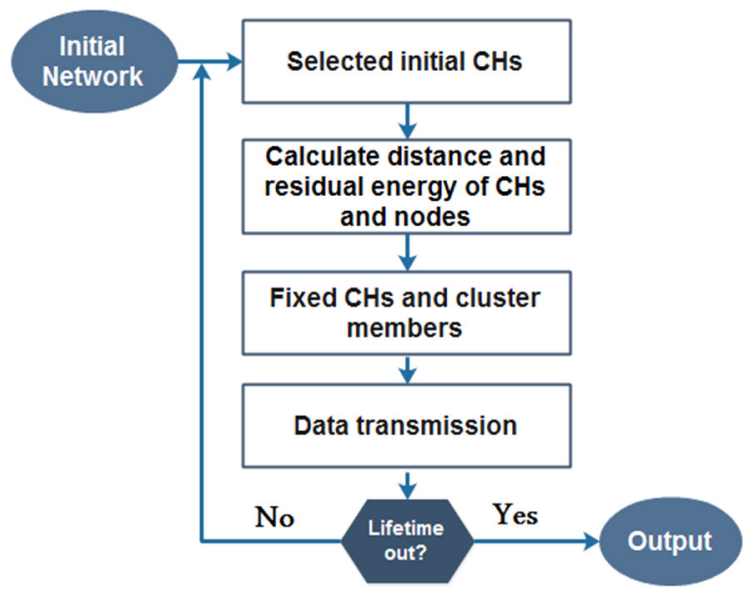

Figure 8 Our proposed clustering algorithm chart flow. 


\section{Simulation}

\subsection{Experimental Setup}

In this work we use NS2 (Network Simulator 2) [30] wich is an object oriented, discrete event driven network simulator targeted at networking research. It provides support of TCP, routing and multicast protocol simulation on all wireless networks. NS2 can be employed in most UNIX systems and Windows. In this paper Ubuntu 14.10 LTS is used as operating system. Most of NS2 process procedure codes are written in $\mathrm{C}++$ (The core of the simulator), $\mathrm{TCL}$ is used as scripting language and Otcl adds object orientation to TCL. The reason for using two programming languages is to have an easy, powerful and fast simulation.

The network model used in this work is as follows: All the sensor nodes in the network are homogeneous, not in mobility, uniformly deployed, and they have the same initial energy. The base station is fixed and located far from the sensor node. The number of cluster heads is $5 \%$ of the number of sensor nodes as in [31]. The rest of simulation parameters are shown in Table 1.

Table 1 Simulation parameters

\begin{tabular}{|l|l|}
\hline \multicolumn{1}{|c|}{ Parameters } & \multicolumn{1}{c|}{ Value } \\
\hline Network size & $250 \times 250 \mathrm{~m}$ \\
\hline Initial energy of node & $100 \mathrm{~J}$ \\
\hline TX, output power & $-5 \mathrm{dBm}$ \\
\hline Eelec & $50 \mathrm{~nJ} / \mathrm{bit}$ \\
\hline Efs & $10 \mathrm{pJ} / \mathrm{bit} / \mathrm{m} 2$ \\
\hline Eamp & $0,0013 \mathrm{pJ} / \mathrm{bit} / \mathrm{m} 4$ \\
\hline EDA & $5 \mathrm{~nJ} / \mathrm{bit}$ \\
\hline $\begin{array}{l}\text { Packet length (number of bits in the packet } \\
\text { sent from CH to BS) }\end{array}$ & 6400 Bits \\
\hline MAC type & Mac/802_11 \\
\hline
\end{tabular}

\section{Simulation results and analysis}

We evaluate the performance of the both protocols LEACH and our proposed algorithm based on K-means using the setup cited above. Results were obtained by means of 10 simulation runs and calculated the average of the measurements.

\subsection{Time taken to cluster}

Both algorithms have the same configuration and the positions of nodes are constant. The time taken is independent of positions of nodes, but it depends only by the variation of nodes numbers and position of cluster head.

We measure the time taken to cluster including time taken to exchanging adverting messages, e.g. time taken to exchanging the position and energy details with all nodes and clustering time and time taken for computing algorithm. In the case of LEACH the time taken to cluster includes also the time taken for the nodes to send back its selection to cluster head. 


\section{Time taken to cluster}

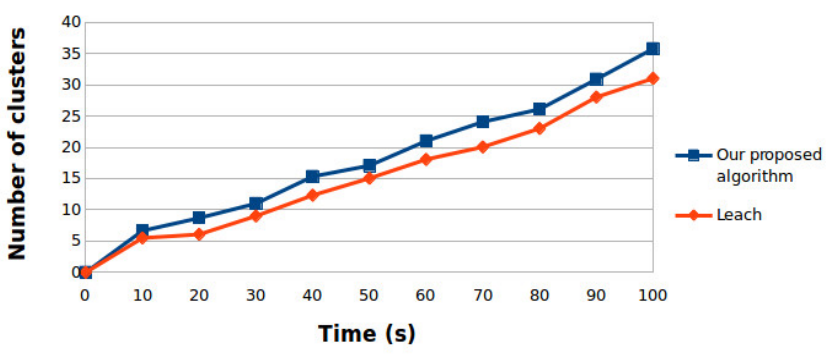

Figure 9 Time taken to form clusters

Analyzing figure 9 we can see that the time taken for LEACH clustering is longer than the time of our proposed clustering algorithm. Probably this is due to the time taken by nodes to send back its selection to cluster head in LEACH algorithm.

\subsection{The average energy}

In general, for clustering, energy is consumed principally for transmitting, receiving packets and for processing. The energy consumed by varying the number of nodes is shown in figure 10.

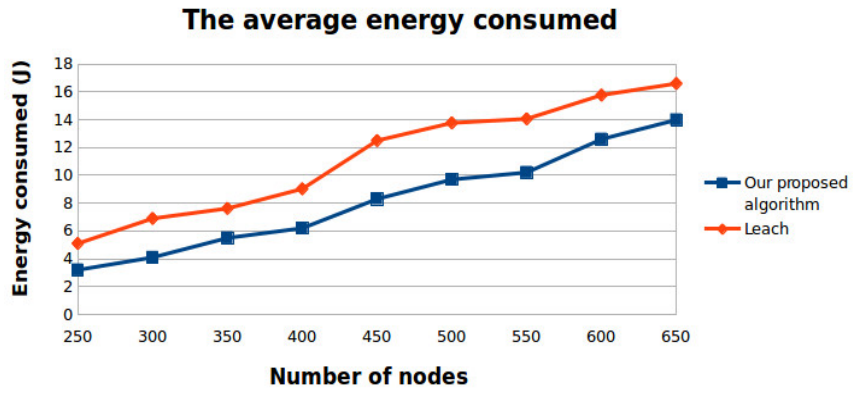

Figure 10 The average energy consumed per node

The average energy consumed per node is measured by computing the difference between the total initial energies of all nodes and total residual energies of all nodes after clustering dividing by the total number of nodes.

Table 2 Energy consumption reduction

\begin{tabular}{|c|c|}
\hline Number of nodes & Energy consumption reduction (\%) \\
\hline 250 & 37.25 \\
\hline 300 & 40.75 \\
\hline 350 & 27.82 \\
\hline 400 & 31.33 \\
\hline 450 & 33.6 \\
\hline 500 & 29.55 \\
\hline 550 & 30.06 \\
\hline 600 & 20.01 \\
\hline 650 & 18.37 \\
\hline
\end{tabular}


Table 2 shows that our proposed algorithm based on k-means reduces the energy consumed compared to $\mathrm{LEACH}$. The percentage of energy consumption reduction is between $19 \%$ and $40 \%$.

\subsection{Network liftime}

The network lifetime is the time until the fraction of alive nodes falls below a predefined threshold or the time during which at least $k$ out of $n$ nodes are alive.

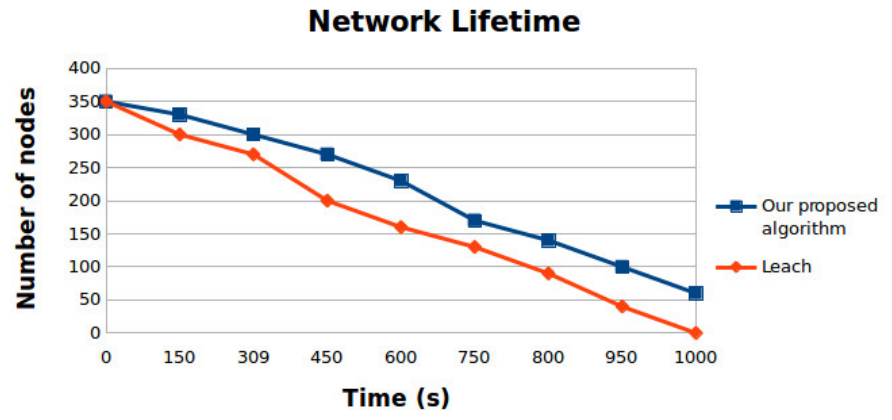

Figure 10 Network lifetime.

As shown in the results of the figure 11 we find that our proposed algorithm based on K-means is more efficient in terms of network lifetime compared to LEACH. Table III illustrates the network lifetime prolonging percentage when using K-means compared to LEACH routing protocol.

Table 3 Nodes Lifetime Prolonging

\begin{tabular}{|c|c|}
\hline Time(s) & Nodes lifetime prolonging (\%) \\
\hline 150 & 10 \\
\hline 300 & 11 \\
\hline 450 & 35 \\
\hline 600 & 43,37 \\
\hline 750 & 30,76 \\
\hline 900 & 55,55 \\
\hline 1050 & 66,66 \\
\hline
\end{tabular}

\subsection{Throughput}

The throughput is depending on the number of packets received by BS.

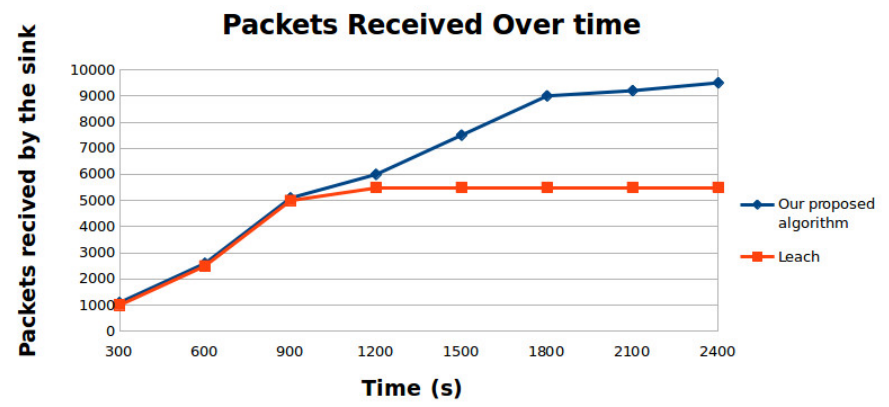

Figure 11 Throughput of LEACH vs our proposed algorithm

We can illustrate from the Figure 12 that the packets received by the BS using our proposed algorithm based on K-means, are considerably superior to LEACH. This improvement can be justified by the fact that 
Hanane Kalkha, Hassan Satori, Khalid Satori. A Dynamic Clustering Approach for Maximizing Scalability in Wireless Sensor Networks. Transactions on Machine Learning and Artificial Intelligence, Vol 5 No 4 August (2017); p: 637-650

when using K-means network lifetime is more extended. Table IV shows the Throughput increasing percentage when using our proposed algorithm compared to LEACH routing protocol.

Table 4 The Throughput Increasing In Case Of K-Means

\begin{tabular}{|c|c|}
\hline Time (s) & $\begin{array}{c}\text { Nodes lifetime prolonging } \\
\text { (\%) }\end{array}$ \\
\hline 300 & 10 \\
\hline 600 & 4 \\
\hline 900 & 7,84 \\
\hline 1200 & 9,09 \\
\hline 1500 & 36,36 \\
\hline 1800 & 70 \\
\hline 2100 & 67,27 \\
\hline 2400 & 72,72 \\
\hline
\end{tabular}

From our simulations, we summarize the obtained results as follows: The throughput received and network life ameliorated, respectably, by $34.66 \%$ and $36.04 \%$ in the case of our proposed algorithm compared to LEACH protocol. Moreover, the average energy consumption decreased by $29.85 \%$.

\section{Conclusion}

Our studies were focused on performance analysis of algorithm based on K-means in WSN. Several scenarios were carried out using different network settings. Our proposed algorithm was compared to a classical algorithm LEACH using the NS2 simulator. The results show that the proposed algorithm offers a considerable energy consumption reduction and extends the life of the network is remarkable and that $\mathrm{K}$ means have the capability to improve the best packet ratio sending by the sensor nodes to the base station. In view of this study, we proposed to extend this work to examine other parameters such as robustness, reachability and QoS, etc. Also, it would be interesting to examine these parameters with other clustering algorithms and to show a comparative study of different clustering methods combined with traditional routing protocols.

\section{REFERENCES}

[1] A. ABBASI and M. YOUNIS, "A survey on clustering algorithms for wireless sensor networks," Computer communications, vol. 30, no 14, pp. 2826-2841, 2007.

[2] F. Akyildiz, W. Su, Y. Sankarasubramaniam, and E. Cayirci, "Wireless sensor networks: a survey," Computer Networks, vol 38, no 4, pp. 393-422, 2002.

[3] Khan, T. Israfil, A. Emdad and A. A. Muhammad, "Multiple Parameter Based Clustering (MPC): Prospective Analysis for Effective Clustering in Wireless Sensor Network (WSN) Using K-Means Algorithm," Wireless Sensor Network, pp. 18-24, no 4, 2012.

[4] M. N. JOSHI, "Parallel k-means algorithm on distributed memory multiprocessors," Computer, vol. 9, 2003. 
Transactions on Machine Learning and Artificial Intelligence Vol 5 No 4, Aug 2017

[5] K. Akkaya, and Y. Mohamed, "A survey on routing protocols for wireless sensor networks," Ad Hoc Networks, no 3, pp. 325-349, 2005.

[6] L, Xu, O. GMP and R Collier, "A Balanced Energy-Efficient Multihop Clustering Scheme for WSN," Wireless and Mobile Networking Conference WMNC, pp. 1-8, May 2014.

[7] X. Liu, "A survey on clustering routing protocols in wireless sensor networks," Sensors, vol. 12, no 8, pp. 11113-11153, 2012.

[8] G. Gupta and M. Younis, "Load-Balanced Clustering in Wireless Sensor Networks," Proceedings of the International Conference on Communication, vol. 3, pp. 1848-1852, Anchorage, Alaska, May 2003.

[9] A. ABABNEH and E. AL-ZBOUN, "EDAC: A Novel Energy-Aware Clustering Algorithm for Wireless Sensor Networks, " International Journal of Advanced Computer Science \& Applications, vol. 1, no 7, pp. 333338, 2016.

[10] W, Heinzelman, A. Chandrakasan and H. Balakrishnan, "Energy-efficient communication protocol for wireless sensor," Proceeding of the Hawaii International,vol. 2, pp.10, Hawaii, January 2000.

[11] R. Kaur, D. Sharma and N. Kaur, "Comparative Analysis Of Leach And Its Descendant Protocols In Wireless Sensor Network," International Journal of P2P Network Trends and Technology, vol. 3, Issue 1, pp. 5155,2013

[12] H. Kalkha, H. Satori and K. Satori, "Performance Analysis of AODV and LEACH Routing Protocol," The International Journal of Multi-disciplinary Sciences - ISSN: 2421-9606, Issue 2 , vol. 2 , pp. 56-60, February - March 2016.

[13] N. Nasri, A. WEI, A. Kachouri and S. E. Khediri, "A New Approach for Clustering in Wireless Sensors Networks," International Workshop on Wireless Networks and Energy Saving Techniques (WNTEST), vol. 32, pp. 1180-1185, Elsevier, 2014.

[14] O. Younis and S. Fahmy, "HEED: a hybrid, energy-efficient, distributed clustering approach for ad hoc sensor networks," IEEE Transactions on Mobile Computing, vol.3, no 4, pp. 366-379, 2004.

[15] S. Lindsey and C. Raghavendra, "PEGASIS: Power-Efficient Gathering in Sensor Information Systems," IEEE Aerospace Conf. Proc 3, pp. 3-1125 - 3-1130, 2002.

[16] K. P. Noufal, "Wireless Sensor Networks - Scalability and Performance Issues: A Review," IJCST, vol. 6, Issue 1, pp. 139-140, Jan - March 2015.

[17] J. N. AL-KARAKI and A. E. KAMAL, "Routing techniques in wireless sensor networks: a survey," IEEE wireless communications, vol. 11, no 6, pp. 6-28.

[18] P. Vipin, S. Girdhari and R. P. YADAV, "Energy efficient clustering scheme for wireless sensor networks: a survey," Journal of Wireless Networking and Communications, vol. 2, no 6, pp. 168-174, 2012.

[19] S. GANESH and R. AMUTHA, "Efficient and secure routing protocol for wireless sensor networks through SNR based dynamic clustering mechanisms," Journal of Communications and Networks, vol. 15, no 4, pp. 422-429, 2013. 
Hanane Kalkha, Hassan Satori, Khalid Satori. A Dynamic Clustering Approach for Maximizing Scalability in Wireless Sensor Networks. Transactions on Machine Learning and Artificial Intelligence, Vol 5 No 4 August (2017); p: 637-650

[20] L. K. Alazzawi, A. M. Elkateeb, A. Ramesh, and W. Aljuhar, "Scalability Analysis for Wireless Sensor Networks Routing Protocols," 22nd International Conference on Advanced Information Networking and Applications, IEEE, pp. 139-144, 2008.

[21] J. H. Chang and L. Tassiulas, "Energy conserving routing in wireless ad hoc networks", Proc. IEEE INFOCOM, pp. 22-31, Mar. 2000.

[22] K. Toh, "Maximum battery life routing to support ubiquitous mobile computing in wireless ad hoc networks", IEEE Communications Magazine, pp. 138-147, June 2001.

[23] M. Blough and P. Santi, "Investigating upper bounds on network lifetime extension for cell-based energy conservation techniques in stationary ad hoc networks," Proceedings of the 8th annual international conference on Mobile computing and networking, ACM, pp. 183-192, 2002.

[24] N. A. Pantazis and S. A. Nikolidakis, "Vergados, Energy-Efficient Routing Protocols in Wireless Sensor Networks: A Survey," IEEE Communications Surveys \& Tutorials, vol. 15, no. 2, pp. 551-591, 2013.

[25] L. ALAZZAWI and ELKATEEB, "Performance evaluation of the WSN routing protocols scalability," Journal of Computer Systems, Networks, and Communications, 2009.

[26] P. Sasikumar, S. Khara, "k-MEANS Clustering in Wireless Sensor Networks", Fourth International Conference on Computational Intelligence and Communication Networks, IEEE, pp. 140-144, 2012.

[27] V. Kawadia, P. R. Kumar, "Power Control and Clustering in Ad Hoc Networks," Proceedings of IEEE INFOCOM. San Francisco, CA, pp. 459-469, March 2003.

[28] J. YICK, B. MUKHERJEE, and D. GHOSAL, "Wireless sensor network survey," Computer networks 52, no. 12, pp. 2292-2330, 2008.

[29] F. ZHAO, Y. XU and R. LI., "Improved LEACH Routing Communication Protocol for a Wireless Sensor Network," International Journal of Distributed Sensor Networks ,2012.

[30] Network Simulator 2: Version 3.35, http://www.isi.edu/nsnam/ns/,, Accessed January 2016.

[31] F. Akyildiz, W. Su, Y. Sankarasubramaniam, and E. Cayirci, "Wireless sensor networks: a survey," Computer networks, vol. 38, no. 4, pp. 393-422, 2002. 